\title{
Similarities of Variant Creutzfeldt-Jakob Disease Strain in Mother and Son in Spain to UK Reference Case
}

\section{Abigail B. Diack, Aileen Boyle, Diane Ritchie, Chris Plinston, Dorothy Kisielewski, Jesús de Pedro-Cuesta, Alberto Rábano, Robert G. Will, ${ }^{1}$ Jean C. Manson'}

We investigated transmission characteristics of variant Creutzfeldt-Jakob disease in a mother and son from Spain. Despite differences in patient age and disease manifestations, we found the same strain properties in these patients as in UK VCJD cases. A single strain of agent appears to be responsible for all vCJD cases to date.

$I^{n}$ n 2008 in Spain, 2 cases of variant Creutzfeldt-Jakob disease (vCJD) in first-degree relatives were identified. After the death of a 41-year-old man (patient 1) from vCJD, his 64-year-old mother (patient 2) began showing symptoms of anxiety and depression and, 2 months later, a gait disorder and progressive dementia. Although the clinical duration was relatively short and the early symptoms uncommon in comparison to vCJD cases in the United Kingdom, the overall clinical phenotype and posterior thalamic hyperintensities as seen in an MRI brain scan led to a diagnosis of suspected vCJD. Neuropathological examination confirmed the diagnosis of vCJD. Both patients were 129MM homozygous, had never received a blood transfusion or tissue graft, and had lived in the same town within the Castilla-León region of Spain (Table 1) (1). The region is a farming area at high risk for bovine spongiform encephalopathy (BSE); 3 of the 5 cases of vCJD reported in Spain came from this region (1). The patients had similar eating habits, which included ingestion of bovine brain. We conducted a study to determine whether these $2 \mathrm{vCJD}$ cases were caused by the BSE agent, whether the agent strain was similar to previously characterized human vCJD cases, and whether the age of the patients would influence the strain characteristics.

\section{The Study}

We challenged cohorts of mice (RIII, C57BL/6J, and $\mathrm{VM}$ ) with frozen central nervous system tissue from the

Author affiliations: The Roslin Institute, Easter Bush, Scotland, UK (A.B. Diack, A. Boyle, C. Plinston, D. Kisielewski, J.C. Manson); University of Edinburgh, Edinburgh, Scotland, UK (D. Ritchie, R.G. Will); Carlos III Institute of Health, Madrid, Spain

(J. de Pedro-Cuesta, A. Rábano)

DOI: https://doi.org/10.3201/eid2309.170159
2 patients from Spain and 1 patient originating from the United Kingdom (Table 1) (2). The Lothian NHS Board Research Ethics Committee provided ethical consent for the use of the UK material for research; the vCJD tissue samples from Spain were provided by NEIKER-Tecnalia (Derio, Spain). We conducted inoculation, clinical scoring, and neuropathological and biochemical analysis of the mice as previously described (3-5). Animal studies were conducted according to the regulations of the UK Home Office Animals (Scientific Procedures) Act 1986.

All 3 vCJD brain isolates transmitted successfully, with the appearance of clinical and pathological signs associated with spongiform encephalopathy transmission (Table 2). Inocula from patients 1 and 2 showed the same temporal order of clinical endpoint in each mouse line when compared with inocula from the UK case (Figure 1). We observed a wide range of incubation periods for each mouse line both within and between inocula (Table 2), which is not unusual in primary transmissions.

We generated vacuolation profiles for each mouse line/inocula combination. In RIII and C57BL/6J mice, we observed moderate to mild vacuolation in the medulla and hypothalamus; C57BL/6J mice also exhibited mild vacuolation in the cerebellar peduncle (Figure 2, panels A, B). VM mice showed mild to moderate vacuolation in the medulla, thalamus, and septum, but typically not in the hypothalamus (Figure 2, panel C). Although the distribution of vacuolation was similar between isolates in the different mouse lines, the intensity of vacuolation distribution varied. This difference was most evident in the VM mice, in which the transmission from patient 2 appeared to have a lower intensity of vacuolation than that of patient 1 and the UK patient.

We observed a widespread accumulation of abnormal prion protein (PrP) throughout the brains of inoculated mice, with greatest accumulations apparent in the medulla, hypothalamus, and thalamic areas. We observed variability in the intensity of PrP accumulation between mice both within and between groups. Fine punctate/punctate deposits were the most common form of PrP accumulation in the mice; however, subtle differences were apparent in the hippocampus. In RIII and C57BL/6J, we observed a characteristic $\operatorname{PrP}$ deposition in the $\mathrm{CA} 2$ region of the hippocampus, whereas VM exhibited PrP deposition in

${ }^{1}$ These senior authors contributed equally to this article. 
Table 1. Demographic and clinical features of 2 case-patients from Spain with variant CJD and reference cases from the United Kingdom*

\begin{tabular}{|c|c|c|c|}
\hline Characteristic & Patient 1 & Patient 2 & UK cases, $n=150$ \\
\hline Patient sex & $\mathrm{M}$ & $\mathrm{F}$ & \\
\hline Case-patient age at illness onset, y & 41 & 64 & 29 (mean) \\
\hline Case-patient age at death, y & 41 & 64 & 30 (mean) \\
\hline Disease duration, mo & 9 & 7 & 14 (mean) \\
\hline Early visual symptoms & + & - & $6 \%$ \\
\hline Early unsteadiness & - & + & $11 \%$ \\
\hline No typical appearance of sporadic CJD on EEG & + & + & $100 \%$ \\
\hline Bilateral symmetric pulvinar high signal on MRI scan of brain & Yes & Yes & $93 \%$ \\
\hline Positive tonsil biopsy result & ND & ND & $19 \%$ \\
\hline History of travel to or residence in United Kingdom & No & No & $100 \%$ \\
\hline Codon 129MM & Yes & Yes & $100 \% \dagger$ \\
\hline Type 2B PrP & Yes & Yes & $100 \% \dagger$ \\
\hline
\end{tabular}

the molecular dentate gyrus with occasional small plaques present in the corpus callosum (online Technical Appendix Figure 1, https://wwwnc.cdc.gov/EID/article/23/9/170159-Techapp1.pdf).

Biochemical analysis of inocula confirmed the presence of protease-resistant prion protein $\left(\mathrm{PrP}^{\mathrm{res}}\right)$ in each of the 3 isolates. We identified a similar $\mathrm{PrP}^{\mathrm{res}}$ type in isolates from the Spain patients that resembled that of the UK patient and the VCJD diagnostic standard, with a banding pattern dominated by the diglycosylated fragment of the protein and an unglycosylated fragment of $\approx 19 \mathrm{kDa}$ (online Technical Appendix Figure 2). PrPres was readily detected in the brain of RIII and VM mice challenged with all 3 isolates. We identified a similar glycosylation pattern in both mouse lines; a dominant diglycosylated fragment of $\approx 30 \mathrm{kDa}$ and an unglycosylated fragment of $\approx 20 \mathrm{k} \mathrm{Da}$. We found no apparent differences between the different mouse line/inocula combinations (online Technical Appendix Figure 3).

\section{Conclusions}

This transmission study of central nervous system tissue from 2 first-degree relatives with vCJD confirms that the same infectious transmissible spongiform encephalopathy
(TSE) agent was responsible for both cases. Comparisons of incubation period, TSE neuropathology, and $\mathrm{PrP}^{\text {res }}$ biochemistry indicate that this strain is consistent with that of a UK case of vCJD and with historical VCJD transmission data (6). The epidemiologic investigation of the 2 related patients indicated that they had shared a common residence and dietary habits, including cattle brain consumption, for $>30$ years. This finding suggests a common source of infection linked to the consumption of highrisk material in a known BSE area, and these transmission studies support the hypothesis that consumption of BSE-contaminated food products is a major risk factor for $\mathrm{vCJD}(7)$.

A feature of the UK vCJD epidemic was the relatively young age of the patients at onset. During 1995-2014, only 6 of 177 cases of vCJD identified in the United Kingdom were in persons $\geq 55$ years of age at the onset of symptoms. Clinical phenotypes in these 6 patients were less consistent than those observed in younger patients (8). The evidence suggests that age is not a barrier to either infection or developing the disease; diagnosis of vCJD may become more important as exposed populations become older. Our study demonstrates that older persons harbor the VCJD agent in the central nervous system in a similar manner to younger

\begin{tabular}{|c|c|c|c|}
\hline \multirow[b]{2}{*}{ Brain inoculum source and mouse line } & \multicolumn{2}{|c|}{ No. mice positive/no. total } & \multirow[b]{2}{*}{ Incubation period, $\mathrm{d}, \pm$ SEM (range) } \\
\hline & Clinical signs of prion disease & Vacuolar pathology & \\
\hline \multicolumn{4}{|c|}{ UK reference case } \\
\hline RIII & $10 / 15$ & $10 / 15$ & $395.3 \pm 17.9(295-489)$ \\
\hline C57BL/6 & $13 / 17$ & $15 / 17$ & $523.7 \pm 19.7(372-637)$ \\
\hline VM & $13 / 16$ & $14 / 16$ & $472.2 \pm 16.1387-552$ \\
\hline \multicolumn{4}{|l|}{ Patient 1} \\
\hline RIII & $14 / 17$ & $15 / 17$ & $417 \pm 14.2(336-516)$ \\
\hline C57BL/6 & $12 / 18$ & $12 / 18$ & $588.4 \pm 25.1(405-706)$ \\
\hline VM & $7 / 18$ & $11 / 18$ & $472.2 \pm 16.1(387-552)$ \\
\hline \multicolumn{4}{|l|}{ Patient 2} \\
\hline RIII & $12 / 16$ & $13 / 16$ & $427.5 \pm 18.4(323-547)$ \\
\hline C57BL/6 & $9 / 18$ & $11 / 18$ & $604.9 \pm 12.4(567-692)$ \\
\hline VM & $4 / 16$ & $7 / 16$ & $524 \pm 16.8(501-573)$ \\
\hline
\end{tabular}

*Bold indicates significant difference $(p<0.05)$ when compared to the mouse line challenged with UK vCJD. vCJD, variant Creutzfeldt-Jakob disease. 


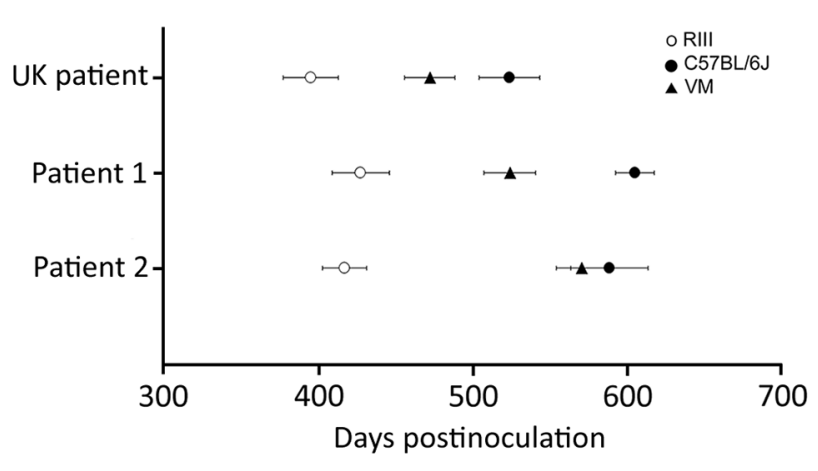

Figure 1. Comparison of VCJD incubation periods in wildtype mice from inoculation of brain tissue homogenates from 2 patients from Spain (son, patient 1; mother, patient 2) with vCJD and a reference patient from the United Kingdom. Results show similar incubation period ranking. Incubation periods were calculated in mice showing clinical and pathologic signs of transmissible spongiform encephalopathy disease. There was a single positive case in VM mice from patient 2. Data show mean incubation period \pm SEM. vCJD, variant CreutzfeldtJakob disease.

persons. Small differences in incubation periods and the intensity of TSE vacuolation are apparent, which may be indicative of variation in the titer of the isolates. It is unknown when the 2 patients from Spain were infected, but if they were exposed at the same time, the 23-year difference in age at time of exposure may have influenced pathogenesis and the ability of the agent to replicate. A delay in neuroinvasion or slower rates of replication in the brain could explain why clinical symptoms are more variable in older patients.

Although our study demonstrates that clinical presentation and infective titer may differ between patients, the overall strain characteristics remain similar. Thus, the vCJD strain can be identified using our strain typing panel regardless of these variable factors.
This study highlights the need for awareness of vCJD in older age groups, particularly in patients with clinical manifestations of atypical dementias. These findings add additional supporting evidence to the hypothesis that a single strain of TSE agent is responsible for vCJD cases, regardless of geographic origin or age at infection, and indirectly support the hypothesis of a dietary origin for primary cases of vCJD.

\section{Acknowledgments}

We thank the Biomedical Research Facility of the Roslin Institute for animal care and the Pathology staff of R(D)SVS for sectioning the mouse brains. We thank Emma Hunt for technical support.

Tissue samples were acquired through the Edinburgh Brain Bank, which is supported by the Medical Research Council (MR/L016400/1).

This report is independent research commissioned and funded by the Department of Health Policy Research Programme (Strain typing of vCJD). The views expressed in this publication are those of the author(s) and not necessarily those of the Department of Health.

Dr. Diack is a Career Track Fellow at the Roslin Institute, University of Edinburgh. Her research interests focus on prion diseases, in particular strain characterization and modeling of human diseases.

\section{References}

1. Riverol M, Palma J-A, Alañá M, Guerrero-Márquez C, Luquin MR, Rábano A. Variant Creutzfeldt-Jakob disease occurring in mother and son. J Neurol Neurosurg Psychiatry. 2012;83:235-6. http://dx.doi.org/10.1136/jnnp.2010.232074

2. Heath CA. Clinical aspect of variant CJD in the UK 1995-2005. University of Aberdeen; 2008.

3. Diack AB, Ritchie D, Bishop M, Pinion V, Brandel JP, Haik S, et al. Constant transmission properties of variant Creutzfeldt-Jakob disease in 5 countries. Emerg Infect Dis. 2012;18:1574-9. http:// dx.doi.org/10.3201/eid1810.120792
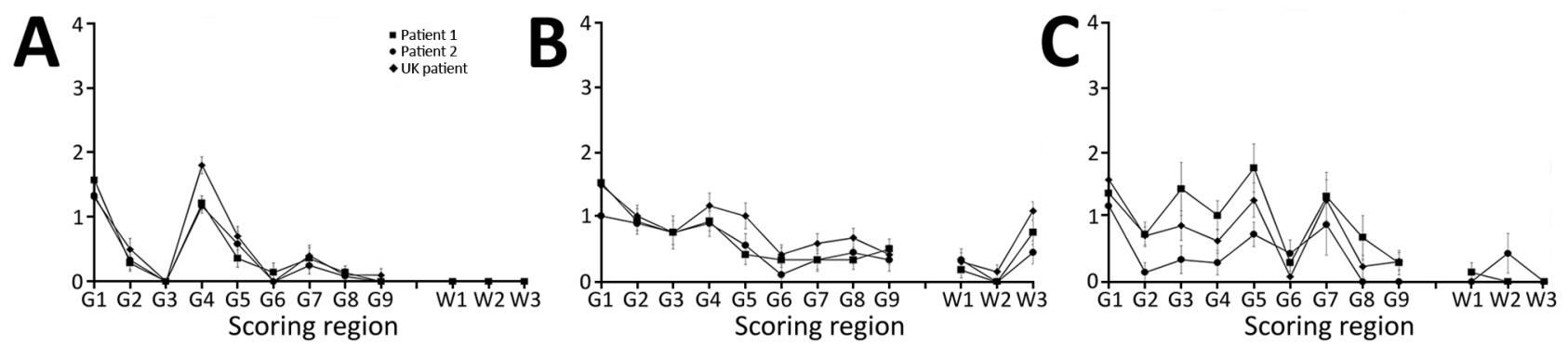

Figure 2. Vacuolation profile of VCJD in wild-type mice from inoculation of brain tissue homogenates from 2 patients from Spain (son, patient 1; mother, patient 2) with vCJD and a reference patient from the United Kingdom. A) RIII mice; B) C57BL/6J mice; C) VM mice. Profiles show similarities in vacuolar pathology intensity and distribution in wild-type mouse brains. Data show mean \pm SEM of clinical and pathologic positive mice, with the exception of VM mice arising from the Spain patients, which also include pathologic positive only mice ( $n \geq 6$ per group). G1-G9, gray matter scoring regions: G1, medulla; G2, cerebellum; G3, superior colliculus; G4, hypothalamus; G5, thalamus; G6, hippocampus; G7, septum; G8, retrosplenial and adjacent motor cortex; G9, cingulate and adjacent motor cortex. W1-W3, white matter scoring regions: W1, cerebellar white matter; W2, mesencephalic tegmentum; W3, pyramidal tract. vCJD, variant Creutzfeldt-Jakob disease. 
4. Dickinson AG, Meikle VMH, Fraser H. Identification of a gene which controls the incubation period of some strains of scrapie agent in mice. J Comp Pathol. 1968;78:293-9. http://dx.doi. org/10.1016/0021-9975(68)90005-4

5. Fraser H, Dickinson AG. The sequential development of the brain lesion of scrapie in three strains of mice. J Comp Pathol. 1968;78:301-11. http://dx.doi.org/10.1016/0021-9975 (68)90006-6

6. Ritchie DL, Boyle A, McConnell I, Head MW, Ironside JW, Bruce ME. Transmissions of variant Creutzfeldt-Jakob disease from brain and lymphoreticular tissue show uniform and conserved bovine spongiform encephalopathy-related phenotypic properties on primary and secondary passage in wild-type mice. J Gen Virol. 2009;90:3075-82. http://dx.doi.org/10.1099/ vir. $0.013227-0$

7. Ward HJ, Everington D, Cousens SN, Smith-Bathgate B, Leitch M, Cooper S, et al. Risk factors for variant CreutzfeldtJakob disease: a case-control study. Ann Neurol. 2006;59:111-20. http://dx.doi.org/10.1002/ana.20708

8. el Tawil S, Mackay G, Davidson L, Summers D, Knight R, Will R. Variant Creutzfeldt-Jakob disease in older patients. J Neurol Neurosurg Psychiatry. 2015;86:1279-80. http://dx.doi.org/10.1136/ jnnp-2014-309397

Address for correspondence: Abigail B. Diack, The Roslin Institute, Easter Bush, Scotland, UK; email: abigail.diack@roslin.ed.ac.uk

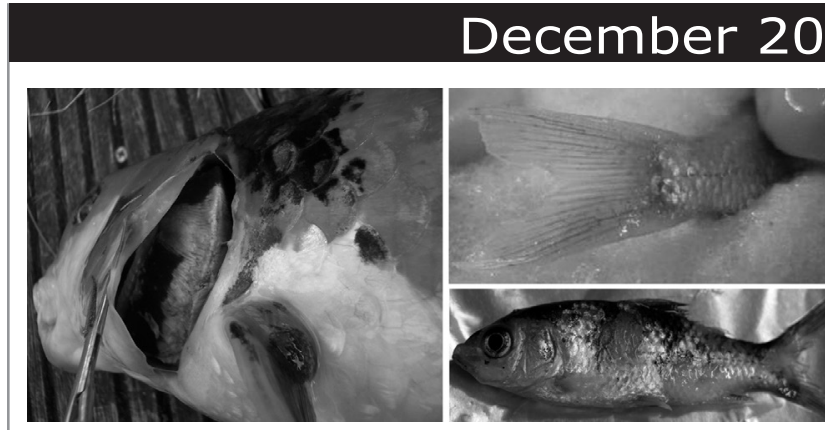

- Surveillance of Wild Birds for Avian Influenza Virus

- Cyprinid Herpesvirus 3

- Reassortant Group A Rotavirus from Straw-colored Fruit Bat

- Hantavirus Pulmonary Syndrome in Argentina, 1995-2008

- Bluetongue Virus Serotype 8

- Epizootic Wave, France, 2007-2008

- Eastern Equine Encephalitis Virus in Mosquitoes and Their Role as Bridge Vectors

- Bartonella spp. in Bats, Kenya

- Alkhurma Hemorrhagic Fever in Humans, Najran, Saudi Arabia

- Environmental Sampling for Avian Influenza Virus A (H5N1) in Live-Bird Markets, Indonesia

- Surveillance and Analysis of Avian Influenza Viruses, Australia

- Freshwater Aquaculture Nurseries and Infection of Fish with Zoonotic Trematodes, Vietnam

- Pandemic (H1N1) 2009 Infection in Patients with Hematologic Malignancy

- Yellow Fever Virus in Haemagogus leucocelaenus and Aedes serratus Mosquitoes, Southern Brazil, 2008
- Reemergence of Rabies in Chhukha District, Bhutan, 2008

- Mortality Risk Factors for Pandemic Influenza on New Zealand Troop Ship, 1918

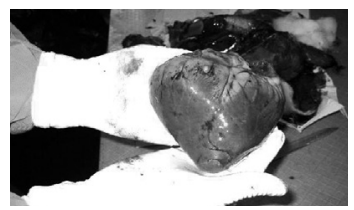

- Oseltamivir-Resistant Pandemic (H1N1) 2009 Virus, South Korea

- Emergence of African Swine Fever Virus, Northwestern Iran

- Mycobacterium tuberculosis Infection of Domesticated Asian Elephants, Thailand

- Hantaviruses and Hantavirus Pulmonary Syndrome, Maranhão, Brazil

- Wild Chimpanzees Infected with 5 Plasmodium Species

- Online Flutracking Survey of Influenza-like Illness during Pandemic (H1N1) 2009, Australia

- Bartonella henselae in Skin Biopsy Specimens of Patients with Cat-Scratch Disease

- Brucella ceti Infection in Harbor Porpoise

- Bundibugyo Ebola Virus Infection, Uganda

- Leishmania tropica Infection in Golden Jackals and Red Foxes, Israel

- Co-detection of Pandemic (H1N1) 2009 Virus and Other Respiratory Pathogens

- Alkhurma Hemorrhagic Fever in Travelers Returning from Egypt

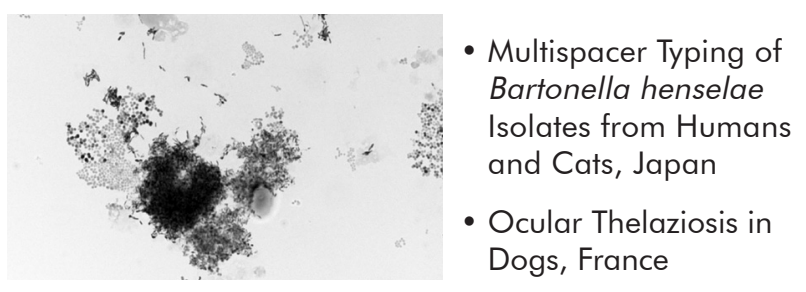

